\title{
Review of: "SPTLC1 p.Leu38Arg, a novel mutation associated with childhood ALS"
}

\author{
Paola Signorelli ${ }^{1}$ \\ 1 University of Milan
}

Potential competing interests: The author(s) declared that no potential competing interests exist.

The present manuscript from Hornemann's group entitled "SPTLC1 p.Leu38Arg, a novel mutation associated with childhood ALS" is an important step forward from their previous published article "Childhood amyotrophic lateral sclerosis caused by excess sphingolipid synthesis". Nat Med 2021. Hornemann's group discovered that amyotrophic lateral syndrome can associate with mutations in the SPTLC1 genes that enhance the rate of sphingoid bases formation. By LCMS, they found an increased amount of dihydrosphingosine and ceramide in patients' serum and in a mutant cellular model. Here the group extends their previous study to a case report: a new ALS mutation, characterized by the same increased sphingolipids synthesis rate. The Authors investigate the mutation-induced alteration of the interaction between SPT1 and ORM3 and conclude that the mutated enzyme lost the negative regulation exerted by ORMs. By investigating the plasma sphingolipids, they found a major increase in dihydroceramide, in line with the enhanced synthesis of LCBs. They also importantly found an increase in the glycosylated ceramides and a modest but significant increase in the deoxy-dihydroceramide. Finally the patient ameliorates upon treatment with a fungal extract that is enriched in myriocin. Patient's blood sphingolipids level is significantly reduced in a two weeks treatment.

In my opinion this is an extremely important and novel set of data, that the science community will take advantage of, and must be published with priority. I have a few questions and recommendations for the Authors.

First. The previous data published on Nature Medicine are not extensively cited here and they are important for any reader in order to appreciate this further step. Therefore, I would state more clearly in the introduction what the Authors already achieved previously. Second, I would strengthen the important findings and step forward of this present work. Not only the modelling of ORM3 and SPT1 interaction, but also the meaning of this sphingolipid flux and the therapeutic approach. For example, the glycolsyl-dhCer and glycosyl-Cer increases found in plasma are not sufficiently commented in the discussion. May these data indicate that glycation is a major metabolic escape systemically, when ceramides species tend to accumulate?

I have a concern on the following sentence, reported in the discussion section: "Increased 1-deoxySL formation was also not seen in the mutant overexpressing HEK cell model, indicating that the SPTLC1-L38R mutation is not per se causing a significant shift in the substrate affinity of the enzyme". I do not fully 
agree with this conclusion and I suggest to use this result to raise a possibility but not a certainty. The in vitro medium supplementation may fully ensure serine demand upon increased SPT activity whereas serine from diet and neo-synthesis in the body may not, ultimately depending on glucose, aminoacids and vitamins intake. The question proposed, if an increase of the LCB synthesis can push towards the use of alanine, is important for basic science and clinical research. Especially because the Authors already proved that serine administration enhances the pathological ALS phenotype. This same question maybe taken into account in several inflammatory conditions in which de novo synthesis of sphingolipids is involved, including type2 diabetes.

Detailed information on where the fungal extract was purchased from, on the preparation method and on concentration used for their experiments must be provided. Moreover, it is highly recommended to show the clinical biochemistry parameters of the patient along with the treatment (in addition to routine evaluations, any parameter that could relate to the lymphocytes status, if available, will be important). Similarly, data on triglycerides, LDL and HDL plasma levels should be provided, before and after fungal treatment.

The Authors assess the activity of the fungal extract on hepatocytes (see below) “The inhibitory effect of the extract on SL de novo synthesis was confirmed in HepG2 hepatocytes, which showed a dose dependent reduction in SPT activity and SL de novo synthesis (Fig. 4B)." This is fine but it must be flanked by the same experiment performed on the ASL cellular model, the SPTLC1 deficient HEK293 cells expressing SPTLC1p.L38R mutant ( the same same line in which sphingolipids increase was measured). Why this experiment was not performed?

Minor comments: In figure 2, please check if DHcer (d18:1) and DHcer(d 18:2) is correct.

The choice of two independent panels of (d18:1) and (d18:2), for the various species analysed can create confusion, as far as no different biological role is assigned to one specie in respect with the other in the manuscript. Given the significant difference in amount of the monounsaturated LCB versus the double unsaturated, for the seek of clarity and simplicity of the take home message of the manuscript, I would suggest to present just one panel with unsaturated LCB and to move the distinction between mono e diunsaturated long chain base in complementary data section. 